Technological University Dublin

DÜBLIN

ARROW@TU Dublin

2008-01-01

\title{
Implementation of Phase-only Modulation Utilizing a Twisted Nematic Liquid Crystal Spatial Light Modulator
}

\author{
Mohammad Mahmud \\ Technological University Dublin \\ Izabela Naydenova \\ Technological University of Dublin, izabela.naydenova@tudublin.ie \\ Vincent Toal \\ Technological University of Dublin, vincent.toal@tudublin.ie
}

Follow this and additional works at: https://arrow.tudublin.ie/cieoart

Part of the Optics Commons

\section{Recommended Citation}

Mahmud, M., Naydenova, I. \& Toal, V. (2008) Implementation of phase-only modulation utilizing a twisted nematic liquid crystal spatial light modulator. Journal of Optics A: Pure and Applied Optics, Vol. 10, No. 8, 10 085007. doi:10.1088/1464-4258/10/8/085007

This Article is brought to you for free and open access by the Centre for Industrial and Engineering Optics at ARROW@TU Dublin. It has been accepted for inclusion in Articles by an authorized administrator of ARROW@TU Dublin. For more information, please contact arrow.admin@tudublin.ie, aisling.coyne@tudublin.ie, gerard.connolly@tudublin.ie.

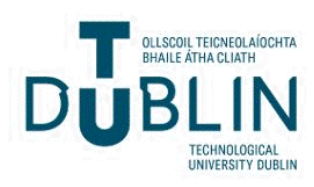




\title{
Implementation of phase-only modulation utilizing a twisted nematic liquid crystal spatial light modulator
}

\author{
Mohammad Sultan Mahmud ${ }^{\mathrm{a}}{ }$, Izabela Naydenova ${ }^{a}$ and Vincent Toal ${ }^{\text {a, }}$ b \\ a) Centre for Industrial and Engineering Optics, Dublin Institute of Technology, Dublin 8, Ireland \\ b) School of Physics, Faculty of Science, Dublin Institute of Technology, Dublin 8, Ireland \\ Tel: ++353 14027927, *Corresponding author's Email: mohammad.mahmudadit.ie
}

\section{Abstract}

Twisted nematic liquid crystal spatial light modulators, (SLM) are widely used for amplitude modulation of light. The technique for measuring phase and amplitude modulation introduced by a twisted nematic cell is not new but the novelty here is that after characterisation of a Holoeye LC2002 TNLC device we were able to identify specific conditions that allow for its use as a pure 180 degree or more, phase modulator with little or no amplitude modulation at $514.5 \mathrm{~nm}$ laser wavelength. The phase shift introduced by the SLM was measured using a Mach-Zehnder interferometer. The experimentally measured and the theoretically predicted intensity transmission and phase shift as functions of gray levels, are compared. Finally, the effects of errors in the angular settings of the polarizers and quarter waveplates on the intensity transmission and phase shift are studied.

\section{Introduction}

Pure phase mode of operation of a SLM can be achieved by careful study of its physical properties - twist angle, $(\alpha)$, input director orientation, maximum (off state) phaseshift, and both voltage-dependent phase shifts due to bulk and edge birefringences ( $\beta$ and $\delta$ ) [1-6]. The intensity transmittance, (T) for a Polarizer-SLM- Analyzer system when the input director is parallel to one of the coordinate axes is expressed as $[5,6]$ :

$$
\mathrm{T}=\left[\mathrm{X}_{1} \cos \left(\varphi_{1}-\varphi_{2}+\alpha\right)+\mathrm{Z} \cdot \sin \left(\varphi_{1}-\varphi_{2}+\alpha\right)\right]^{2}+\left[\mathrm{Y}_{1} \cdot \cos \left(\varphi_{1}+\varphi_{2}-\alpha\right)\right]^{2}
$$

where,

$$
\mathrm{X}_{1}=\cos \gamma \cdot \cos 2 \delta-\frac{\beta}{\gamma} \sin \gamma \cdot \sin 2 \delta ; \mathrm{Y}_{1}=\cos \gamma \cdot \cos 2 \delta+\frac{\beta}{\gamma} \sin \gamma \cdot \cos 2 \delta ; \mathrm{Z}=\frac{\alpha}{\gamma} \sin \gamma, \gamma=\sqrt{\alpha^{2}+\beta^{2}}
$$


Here, $\varphi_{1}$ and $\varphi_{2}$ are the polarizer and analyzer angles respectively, measured with respect to the input director of the SLM Here, all angles are taken as positive when measured clockwise looking from the detector side and the transmissions are normalized in order to eliminate the intensity fluctuations [7]. By using the Solver fitting utility of Excel at three experimental configurations $\left(\varphi_{1}=0, \varphi_{2}=\alpha+90^{\circ}\right),\left(\varphi_{1}=45^{\circ}, \varphi_{2}=\alpha-45^{\circ}\right),\left(\varphi_{1}=22.5^{\circ}, \varphi_{2}=\alpha+112.5^{\circ}\right)$ and at four wavelengths the values of $\beta(V)$ and $\delta(V)$ as functions of gray level $[5,6]$ were obtained. It was then possible to search for any desired modulation behaviour by introducing external polarization components. The most general way to obtain any desired amplitude or phase modulation behaviour is to use a polarizer-QWP-SLM-QWP-analyzer setup $[6,8]$. This setup was used to generate a desired elliptically polarized eigenvector whose ellipticity changes as a function of the birefringence of the LC which in turn is proportional to the applied voltage [9]. The intensity transmittance and total phaseshift for such a system are given by [6]:

$$
\begin{aligned}
& \mathrm{T}=\mathrm{ARE}^{2}+\mathrm{AMM}^{2} \\
& \psi=-\beta-2 . \delta+\tan ^{-1}\left(\frac{\mathrm{AMM}}{\mathrm{ARE}}\right) .
\end{aligned}
$$

where, $A_{R E}$ and $A_{\mathrm{LM}}$ are the real and imaginary parts of the whole system with

$$
\begin{aligned}
& A_{28}=X_{1}\left\{\cos ^{2} \Phi \cdot \cos \left(\varphi_{1}-\varphi_{2}+\alpha\right)-\sin ^{2} \Phi \cdot \cos \left[2\left(\eta_{1}-\eta_{2}\right)-\varphi_{1}+\varphi_{2}+\alpha\right]\right\} \\
& -Y_{1} \cos \Phi \cdot \sin \Phi \cdot\left\{\cos \left[2 \eta_{2}+\varphi_{1}-\varphi_{2}-\alpha\right]+\cos \left[2 \eta_{1}-\varphi_{1}+\varphi_{2}-\alpha\right]\right\} \\
& +Z_{1}\left\{\cos ^{2} \Phi \cdot \sin \left(\varphi_{1}-\varphi_{2}+\alpha\right]-\sin ^{2} \Phi \cdot \sin \left[2\left(\eta_{1}-\eta_{2}\right)-\varphi_{1}+\varphi_{2}+\alpha\right]\right\} \text {. } \\
& \mathrm{A}_{\mathrm{M}}=-\mathrm{X}_{1} \cos \Phi \sin \Phi\left\{\cos \left(2 \eta_{2}-\varphi_{1}-\varphi_{2}-\alpha\right)+\cos \left[2 \eta_{1}-\varphi_{1}-\varphi_{2}+\alpha\right]\right\} \\
& +Y_{1}\left\{-\cos ^{2} \Phi \cdot \cos \left(\phi_{1}+\phi_{1}-\alpha\right)+\sin ^{2} \Phi \cdot \cos \left[2\left(\eta 1+\eta^{2}\right)-\phi_{1}-\phi_{2}-\alpha\right]\right\} \\
& +Z \cos \Phi \cdot \sin \Phi\left\{\sin \left[2 \eta_{2}-\varphi_{1}-\varphi_{2}-\alpha\right]-\sin \left[2 \eta_{1}-\varphi_{1}-\varphi_{2}+\alpha\right]\right\} \text {. }
\end{aligned}
$$

Here $\eta_{1}, \eta_{2}$ are the orientations of the slow axes of the first and second QWPs. The value of $\Phi$ is $45^{\circ}$ fixed by the QWPs at the desired $514.5 \mathrm{~nm}$ wavelength.

In order to evaluate the influence of the misalignment of the angular positions of the polarising components on the output light properties (transmitted intensity and phase) equation (2) and (3) must be analysed by differentiation. Transmission and phase errors, $(\Delta \mathrm{T}, \Delta \psi)$ due to small angular 
misalignments of polarizer, $\left(\Delta \varphi_{1}\right)$, analyzer, $\left(\Delta \varphi_{2}\right)$ and first and second QWPs $\left(\Delta \eta_{1}, \Delta \eta_{1}\right)$ are given by

$$
\Delta \mathrm{T}_{\varphi 1}=\frac{\partial \mathrm{T}}{\partial \varphi_{1}} \cdot \Delta \varphi_{1} ; \Delta \mathrm{T}_{\varphi_{2}}=\frac{\partial \mathrm{T}}{\partial \varphi_{2}} \cdot \Delta \varphi_{2} ; \Delta \mathrm{T}_{\eta 1}=\frac{\partial \mathrm{T}}{\partial \eta_{1}} \cdot \Delta \eta_{1} ; \Delta \mathrm{T}_{\eta_{2}}=\frac{\partial \mathrm{T}}{\partial \eta_{2}} \cdot \Delta \eta_{2}
$$

and,

$$
\Delta \psi_{\varphi 1}=\frac{\partial \psi}{\partial \varphi_{1}} \Delta \varphi_{1} ; \Delta \psi_{\varphi_{2}}=\frac{\partial \psi}{\partial \varphi_{2}} \Delta \varphi_{2} ; \Delta \psi_{\eta_{1}}=\frac{\partial \psi}{\partial \eta_{1}} \Delta \eta_{1} ; \Delta \psi_{\eta_{2}}=\frac{\partial \psi}{\partial \eta_{2}} \cdot \Delta \eta_{2}
$$

where,

$$
\begin{aligned}
& \frac{\partial T}{\partial \varphi_{1}}=2\left[A_{R E}-\frac{\partial A_{R E}}{\partial \varphi_{1}}+A_{I M} \cdot \frac{\partial A_{I M}}{\partial \varphi_{1}}\right] ; \quad \frac{\partial T}{\partial \varphi_{2}}=2\left[A_{R E} \cdot \frac{\partial A_{R E}}{\partial \varphi_{2}}+A_{M M} \cdot \frac{\partial A_{M}}{\partial \varphi_{2}}\right] \\
& \frac{\partial \mathrm{T}}{\partial \eta_{1}}=2\left[\mathrm{~A}_{\mathrm{RE}} \cdot \frac{\partial \mathrm{A}_{\mathrm{RE}}}{\partial \eta_{1}}+\mathrm{A}_{\mathrm{IM}} \cdot \frac{\partial \mathrm{A}_{\mathrm{MM}}}{\partial \eta_{1}}\right] ; \quad \frac{\partial \mathrm{T}}{\partial \eta_{2}}=2\left[\mathrm{~A}_{\mathrm{RE}} \cdot \frac{\partial \mathrm{A}_{\mathrm{RE}}}{\partial \eta_{2}}+\mathrm{A}_{\mathrm{MM}} \cdot \frac{\partial \mathrm{A}_{\mathrm{M}}}{\partial \eta_{2}}\right]
\end{aligned}
$$

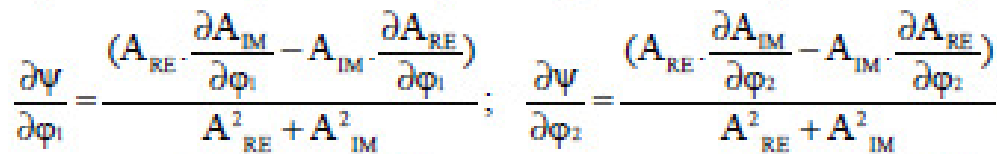

$$
\begin{aligned}
& \frac{\partial \psi}{\partial \eta_{1}}=\frac{\left(\mathrm{A}_{\mathrm{RE}} \cdot \frac{\partial \mathrm{A}_{\mathrm{MM}}}{\partial \eta_{1}}-\mathrm{A}_{\mathrm{MM}} \cdot \frac{\partial \mathrm{A}_{\mathrm{RE}}}{\partial \eta_{1}}\right)}{\mathrm{A}_{\mathrm{RE}}^{2}+\mathrm{A}_{\mathrm{M}}^{2}} \text {; and } \frac{\partial \mathrm{T}}{\partial \eta_{2}}=\frac{\left(\mathrm{A}_{\mathrm{RE}} \cdot \frac{\partial \mathrm{A}_{\mathrm{MM}}}{\partial \eta_{2}}-\mathrm{A}_{\mathrm{MM}} \cdot \frac{\partial \mathrm{A}_{\mathrm{RE}}}{\partial \eta_{2}}\right)}{\mathrm{A}_{\mathrm{RE}}^{2}+\mathrm{A}_{\mathrm{MM}}^{2}}
\end{aligned}
$$

The theoretical values for $\Delta \mathrm{T}$ and $\Delta \psi$ can be calculated by calculation of the partial differentials $(5 \mathrm{~A})$ and $(5 \mathrm{~B})$ for specific values of the misalignments in the angular positions of the each elements - $\Delta \varphi_{1}, \Delta \varphi_{2}, \Delta \eta_{1}$ and $\Delta \eta_{2}$. For practical reasons this deviations should not exceed $\pm 2^{\circ}$.

The total transmission and phase errors, $(\delta \mathrm{T}, \delta \psi)$ due to error in positioning of all the elements in the setup can be found as

$$
\delta \mathrm{T}=\sqrt{\left(\left|\frac{\partial T}{\partial \varphi_{1}}\right| \cdot \Delta \varphi_{1}\right)^{2}+\left(\left|\frac{\partial T}{\partial \varphi_{2}}\right| \cdot \Delta \varphi_{2}\right)^{2}+\left(\left|\frac{\partial T}{\partial \eta_{1}}\right| \cdot \Delta \eta_{1}\right)^{2}+\left(\left|\frac{\partial T}{\partial \eta_{2}}\right| \cdot \Delta \eta_{2}\right)^{2}}
$$

and,

$$
\delta \psi=\sqrt{\left(\left|\frac{\partial \psi}{\partial \varphi_{1}}\right| \cdot \Delta \varphi_{1}\right)^{2}+\left(\left|\frac{\partial \psi}{\partial \varphi_{2}}\right| \Delta \varphi_{2}\right)^{2}+\left(\left|\frac{\partial \psi}{\partial \eta_{1}}\right| \cdot \Delta \eta_{1}\right)^{2}+\left(\left|\frac{\partial \psi}{\partial \eta_{2}}\right| \cdot \Delta \eta_{2}\right)^{2}}
$$

\section{Experimental Methods for measuring phase shift and intensity modulation}

The Mach-Zehnder interferometer is commonly used for measuring the phase shift introduced after transmission through a SLM [10-14]. Zhang [15] and McClain [16] used diffraction 
techniques for measuring phase shift, which are noninterferometric but have the disadvantage of being derived from intensity measurements, as higher measurement accuracy depends upon camera resolution and quantization by the image board. Marquez [17] used diffraction gratings for measuring the phase shift experimentally, which required very precise Ronchi components, in order to obtain required equal intensities in the first orders. We used a near common-path MachZehnder interferometer (Fig.1) to measure phase shift because this technique is less sensitive to temperature, vibration, and is simple but cost effective as the SLM is effectively split into two parts. An ordinary CCD camera or a webcam could be used to capture changes in the fringe pattern for measuring phase shift. A laser beam was expanded and collimated to illuminate the SLM through a double-hole mask in front of the SLM, separating it into two distinct parts; one part is addressed with a constant gray level of 0 , whereas the other part is addressed with gray levels from 0 to 255 . The holes had to be identical to obtain the best contrast in the fringe pattern.

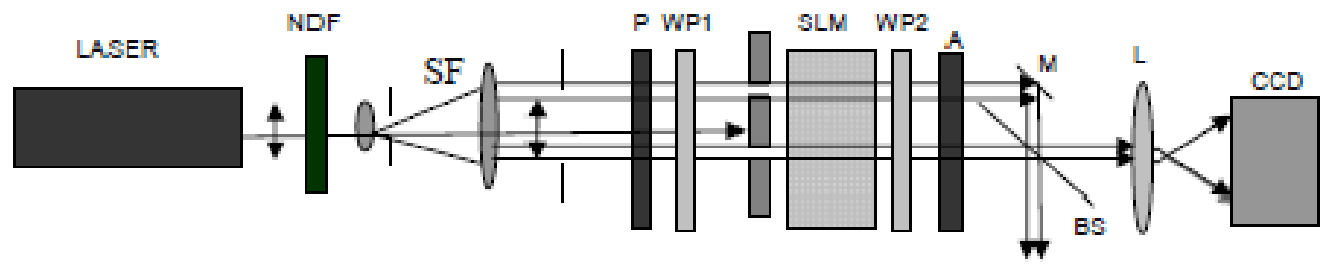

Fig 1: Experimental setup for measuring phase shift. Here, NDF: neutral Density filter, SF: spatial filter, $\mathbb{P}$ : polarizer, WP: quarter wave plate, A: analyzer, SLM: spatial light modulator, $\mathrm{M}$ : mirror, BS: beam Splitter, and L- lens.

It is a usual practice to use a 4-f system [18] to enable the beams to interfere with each other, (for example, Holoeye used this technique), but in our experiment an ordinary beam splitter behind the SLM was used to overlap the two beams [Fig.1]. A lens (L) was used to expand the interference pattern onto a webcam (Pro 5000, Logitech). The phase shift appeared as a shift in the interference pattem and was evaluated by the 'Phase cam' software [18]. A program written in LabVIEW was used to change the gray level of the SLM in order to measure the intensity modulation. Intensity modulation was measured by blocking one of the splitting beams and replacing the webcam with a photo detector and the whole display was addressed with a homogeneous gray level. 


\section{Results}

\subsection{Characterisation of the basic properties of the SLM}

By using the Solver utility of Excel module at three experimental configurations $\left(\varphi_{1}=0, \varphi_{2}=\right.$ $\left.\alpha+90^{\circ}\right),\left(\varphi_{1}=45^{\circ}, \varphi_{2}=\alpha-45^{\circ}\right),\left(\varphi_{1}=22.5^{\circ}, \varphi_{2}=\alpha+112.5^{\circ}\right)$ and at four wavelengths, the values of $\beta(V)$ and $\delta(V)$ as functions of gray level, were found [5, 6] [Fig.2]. Once $\beta(V)$ and $\delta(V)$ were obtained, we used the polarizer-QWP1-SLM-QWP2-analyzer setup to get the desired phase-only or amplitude modulation.

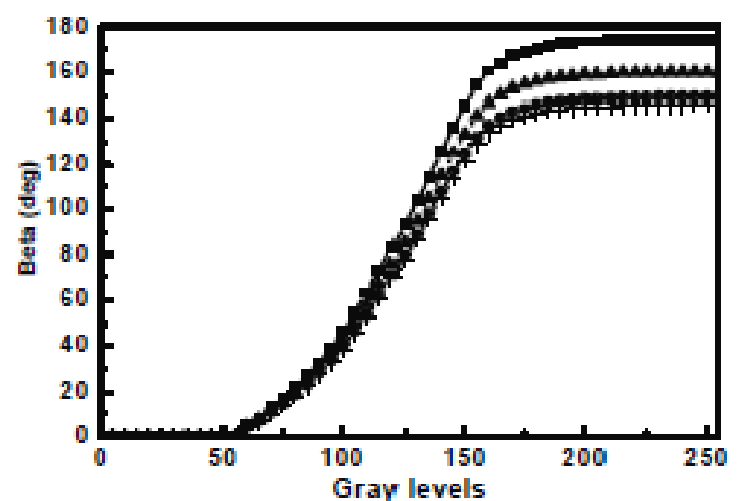

(a)

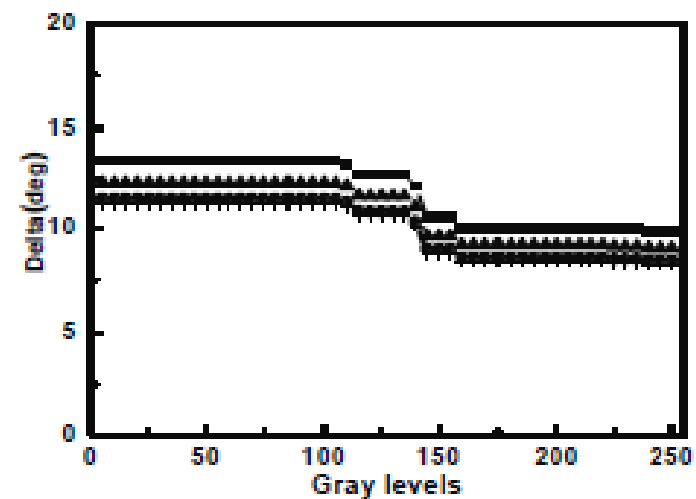

(b)

Fig.2: Phase shifts due to (a) Bulk birefringence, $\beta(V)$ and (b).Edge birefringences, $\delta(V)$ at four

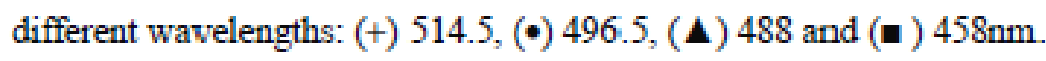

\subsection{Polariser-QWP-SLM-analyser set up}

In this study we used a polarizer-QWP1-SLM-analyzer setup to get the desired phase-only or amplitude modulation. To simplify the setup, three parameters, $\left(\varphi_{1}, \eta_{1} \varphi_{2},\right)$ were taken as free parameters for Solver searching and a maximum of $182^{\circ}$ phase shift was found with nearly flat intensity modulation over the entire gray level range. The required orientation angles of the polarizer, analyzer, and QWP1 were found to be $-14.24^{\circ},-33.42^{\circ}$, and $6.84^{\circ}$ respectively with respect to the input director of the SLM. The theoretical intensity modulation was almost flat, having a variation of $6 \%$ over the entire range of gray levels. 


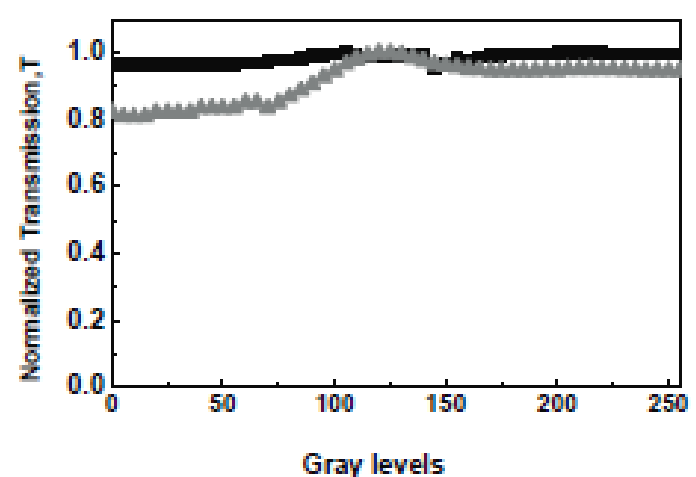

(a)

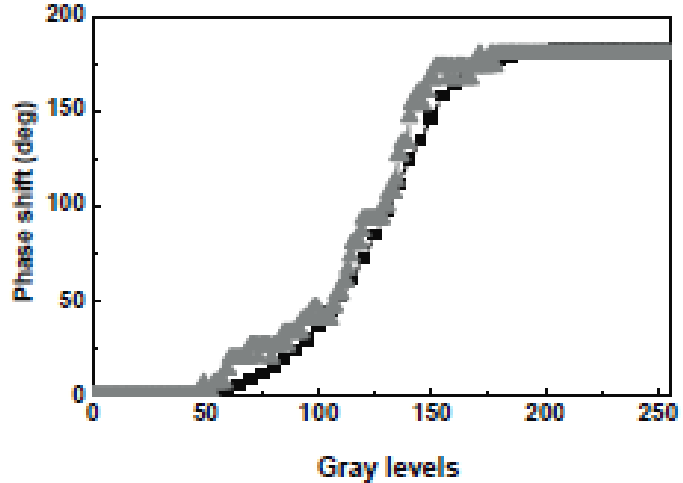

(b)

Fig.3: Theoretical (a) and experimental ( $\Delta$ ) transmission (a) and phase shift (b) at $514.5 \mathrm{~nm}$ for 'P-QWP1-SLM-A' setup.

From figure 3(a) it is seen that the experimental intensity modulation is slightly different from the theoretical results. To investigate the influences of angular misalignment of the optical components, the analyzer, polarizer and the quarter wave plate angular positions were deliberately changed by up to $\pm 2^{\circ}$ from their theoretically determined values. Measurement were carried out when two of the three polarizing elements were kept constant at their theoretically predicted values using the Solver method and only one of them was misaligned by $\pm 2^{0}$ in order to analyze misalignment errors. The experimental results showed that significant changes in the transmission occurred even with small changes of these angles (Fig.4). The influence of the misalignment in the position of the analyzer is shown in Fig.4a. It is seen that deviation of the analyzer's angle by $\pm 2^{\circ}$ from the theoretically predicted value of $-33.42^{\circ}$ would lead to more than $10 \%$ deviation in the transmission especially at low gray levels. Similar results were observed for the misalignment in the polarizer's position (Fig.4b) and the QWP1 position (Fig.4c). Therefore, the slight disagreement between the theoretically predicted and the experimentally measured values for the SLM transmission (Fig 3a) could be due to small errors in the misalignment of the optical components. At the same time, measurements of the phase of the transmitted through the SLM light when the angular positions of the polarising elements are varied within $\pm 2^{\circ}$ from the theoretically predicted values show that the phase varies $1 \%$ from the expected value. 


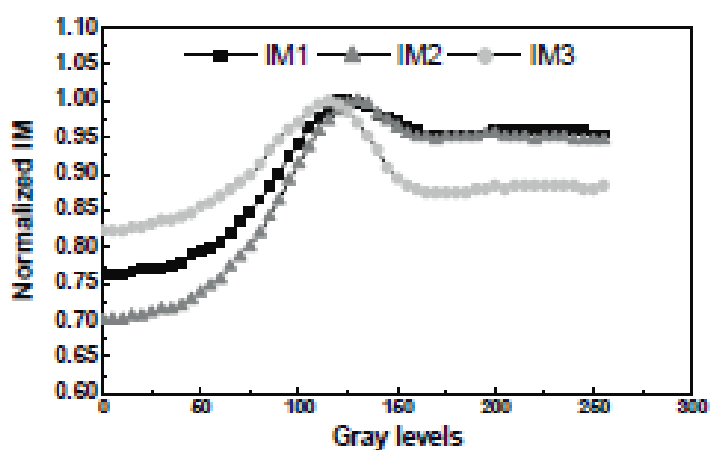

(a)

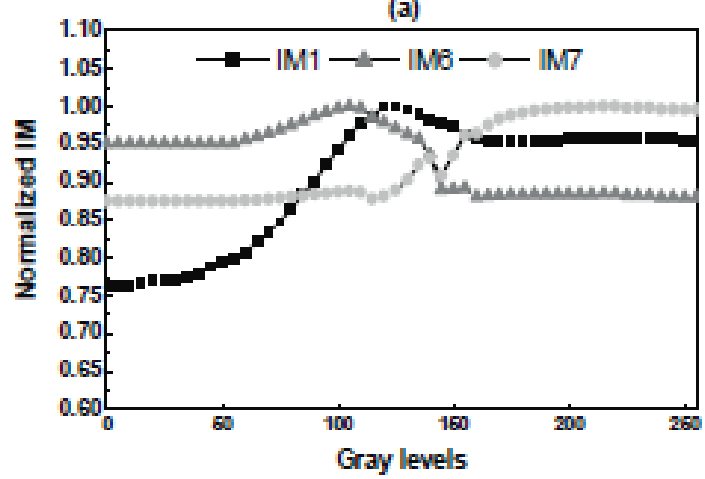

(c)

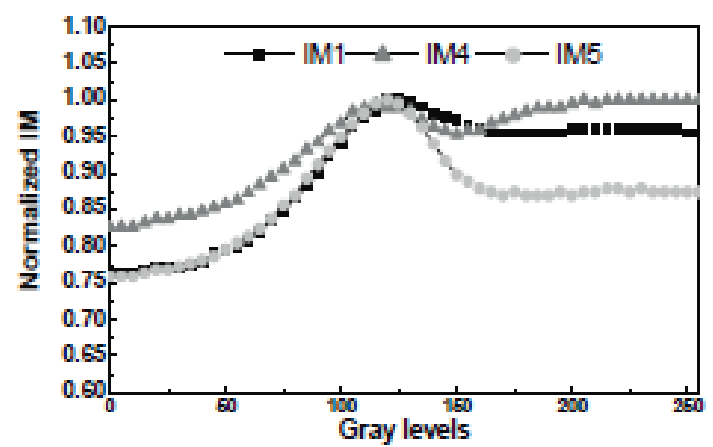

(b)

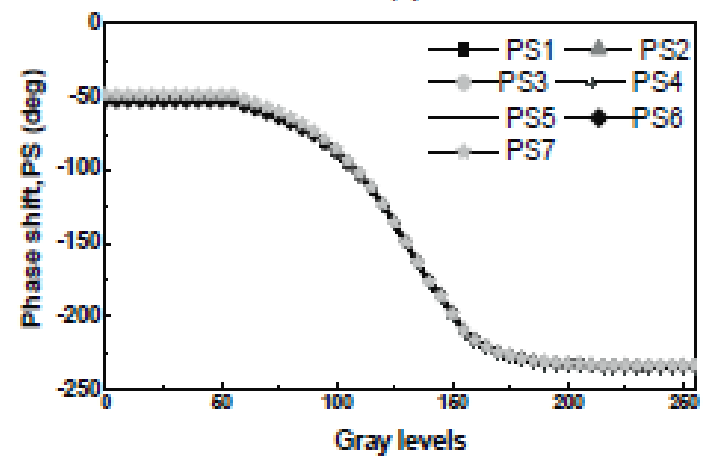

(d)

Fig.4: Experimental intensity transmission due to \pm 2 degree misalignment of angles of (a) analyzer, (b) polarizer, and (c) QWP and (d) their corresponding phase shifts, (PS) for P-QWPSLM-A setup. Here the angles of polarizer, QWP and analyzer are IM1 $\left(-14.24^{\circ}, 6.84^{\circ},-33.42^{\circ}\right)$, $\operatorname{IM} 2\left(-14.24^{\circ}, 6.84^{0},-35.42^{\circ}\right), \operatorname{IM} 3\left(-14.24^{\circ}, 6.84^{\circ},-31.42^{\circ}\right), \operatorname{IM} 4\left(-16.24^{0}, 6.84^{\circ},-33.42^{\circ}\right), \operatorname{IM} 5(-$ $\left.12.24^{\circ}, 6.84^{\circ},-33.42^{\circ}\right), \operatorname{IM} 6\left(-14.24^{\circ}, 4.84^{\circ},-33.42^{\circ}\right)$, and $\operatorname{IM} 7\left(-14.24^{\circ}, 8.84^{\circ},-33.42^{\circ}\right)$ respectively.

\subsection{Polariser-QWP1-SLM-QWV2-analyser setup}

The best match for constant intensity modulation and more than $180^{\circ}$ phase shift was found when all parameters, ( $\varphi_{1}, \eta_{1} \varphi_{2}$, and $\left.\eta_{2}\right)$ were taken as free parameters during computer searching and the values of $\varphi_{1}, \eta_{1} \varphi_{2}$, and $\eta_{2}$ were found to be $144^{\circ}, 3.3^{\circ},-48.25^{\circ}$ and $-88.78^{\circ}$ respectively from the input director looking from the detector side. The theoretical and experimental intensity transmission and phase shift agree with each other [Fig. 5] and a phase shift up to $224^{\circ}$ (Fig.5b) was achieved at laser wavelength $514.5 \mathrm{~nm}$. One conclusion from this result is that pure phase modulation of $180^{\circ}$ could be achieved at even longer wavelength using this SLM. For these angular settings of the polarizing components the theoretically predicted intensity varies within $3 \%$ over the entire range of gray levels from flat level. The experimentally measured variation 
was within ...\% which is in a good agreement with the theoretically predicted dependence on the gray level (Fig.5a).

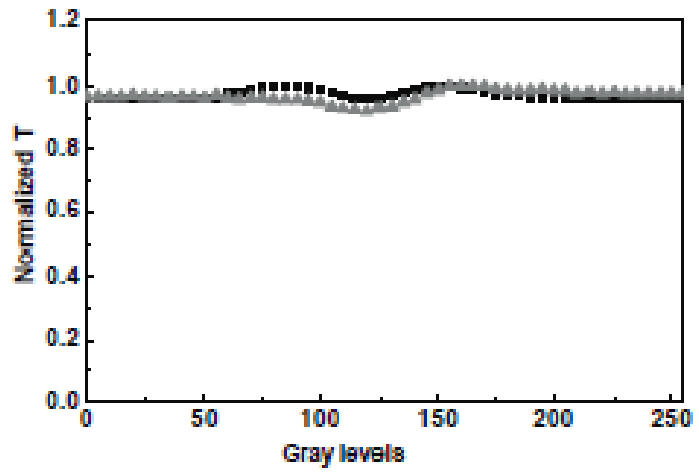

(a)

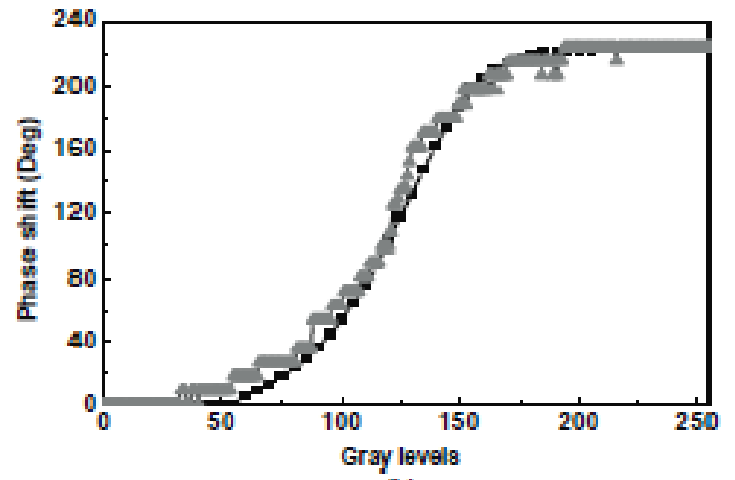

(b)

Fig. 5: Theoretical (a) and experimental ( $\Delta$ ) transmission (a) and phase shift (b) at $514.5 \mathrm{~nm}$ for P-QWP1-SLM-QWP2-A setup.

\subsection{Theoretical study of the transmission and phase shift errors due to misalignment of the polarizing components}

To investigate transmission and phase shift error, $(\Delta \mathrm{T}$, and $\Delta \psi)$ due to the influences of angular misalignment of polarizers and both QWPs, their angular positions were deliberately changed $\pm 2^{\circ}$ from their theoretical values. These errors were analyzed using equation $5 \mathrm{C}$, where, $\Delta \varphi_{1}, \Delta \varphi_{2} \Delta \eta_{1}$, and $\Delta \eta_{2}$ are the misalignment errors. The results showed that when both QWPs and polarizers were taken as free parameters during Solver searching, $\pm 2^{\circ}$ changes of the angle do not affect transmission significantly. The maximum variation in transmittance is $2.5 \%$ [Fig.6a] and in phase shift the maximum variation is 3 degrees [Fig.6b], whereas, when one QWP and polarizer angles were taken as free parameters then maximum variation of transmittance is $12 \%$ [Fig. 7a] and the phase shift variance is 5 degrees [Fig 7b]. Therefore the, 'P-QWP1-SLM-QWP2-A' setup is more stable against small errors in the positions of the polarising components. 


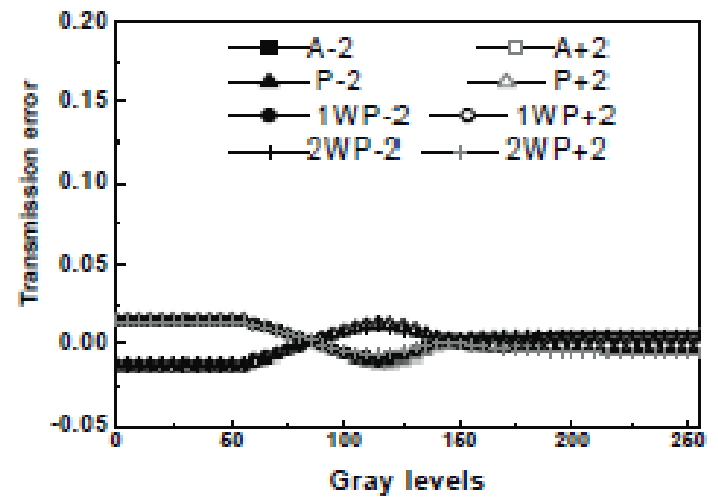

(a)

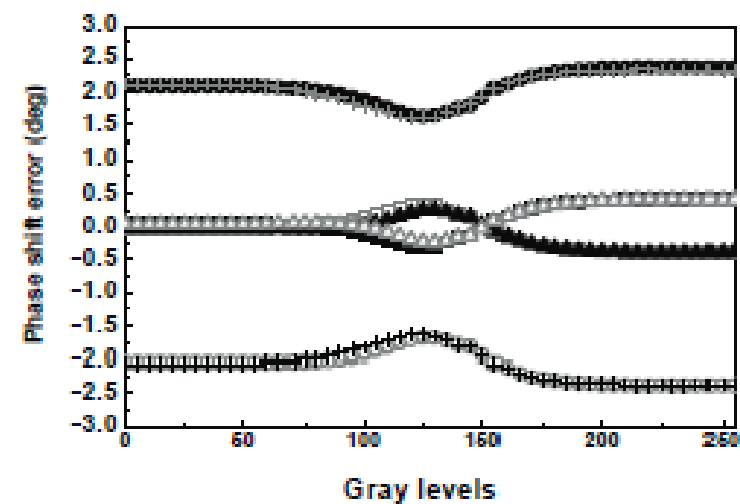

(b)

Fig. 6: Theoretically calculated transmission error, $(\Delta \mathrm{T})$ (a) and phase error, $(\Delta \psi)$ (b) due to \pm 2 degree misalignment of each optical components for P-QWP-SLM-QWP-A setup. Each graph represents the misalignment of one component: analyser (squares), polariser (triangles), QWP1 (circles) and QWP2 (crosses) while the positions of the other polarising components remain unchanged and their angles are equal to the theoretically predicted by the Solver method values which are $144^{\circ}$ for the analyser, $3.3^{\circ}$ for the polariser, $-48.25^{\circ}$ for QWP1 and $-88.78^{\circ}$ for QWP2.

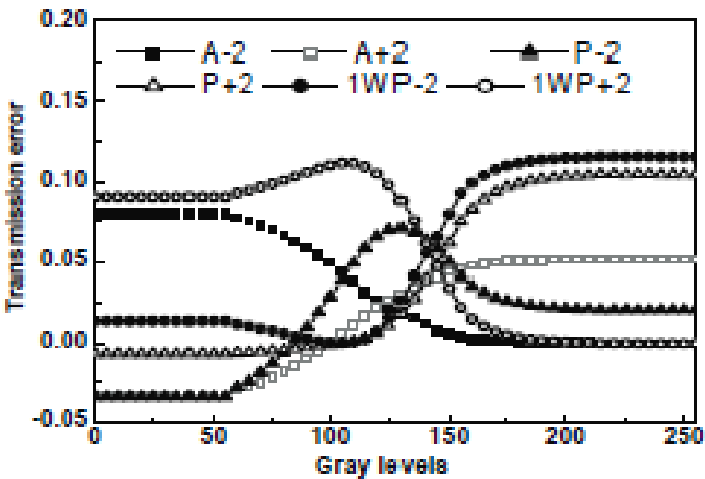

(a)

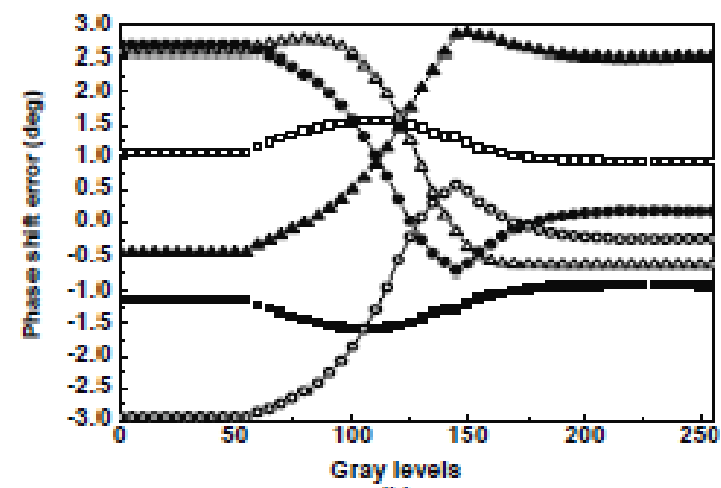

(b)

Fig. 7: Theoretically calculated transmission error, $(\Delta \mathrm{T})$ (a) and phase error, $(\Delta \psi)$ (b) due to \pm 2 degree misalignment of each optical components for P-QWP1-SLM-A setup. Each graph represents the misalignment of one component: analyser (squares), polariser (triangles), QWP1 (circles) while the positions of the other polarising components remain unchanged and their angles are equal to the theoretically predicted by the Solver method values which are $-14.24^{\circ}$ for the analyser, $-33.42^{\circ}$,for the polariser and $6.84^{\circ}$ for the QWP1.

In order to estimate the total transmission and phase error, $(\delta \mathrm{T}, \delta \psi)$ due to simultaneous: misalignment of 2 degrees of all polarising components the equations $6 \mathrm{~A}$ and $6 \mathrm{~B}$ were used for 
the P-QWP1-SLM- A' and P-QWP1-SLM-QWP2-A' systems. The results for the total error in transmission, $(\delta \mathrm{T})$ and phase, $(\delta \psi)$ are shown in Fig. $8 \mathrm{a}$ and Fig. $8 \mathrm{~b}$ respectively. It is seen from these two figures that the "P-QWP1-SLM-QWP2-A' set up is characterised by smaller error in transmission and phase than the P-QWP1-SLM -A' set-up.

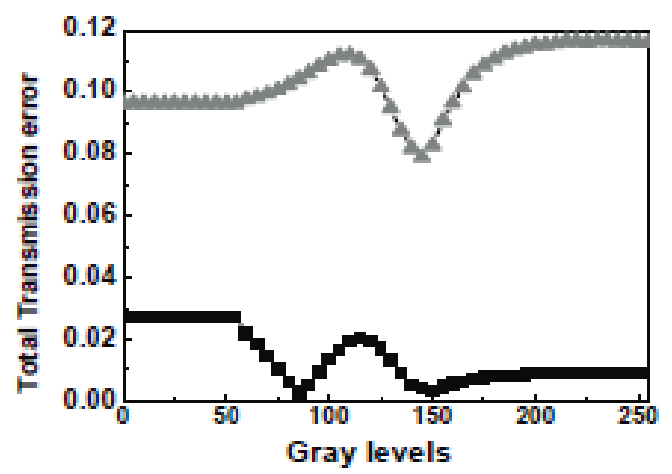

(a)

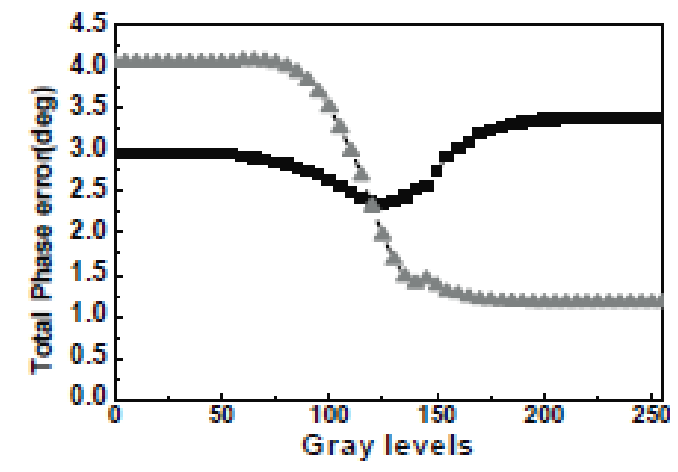

(b)

Fig8. Theoretically calculated total error in transmittance (a) and in phase (b) due to 2 degrees misalignment of all polarising components for the P-QWP1-SLM-QWP2-A system (u) and the P-QWP1-SLM-A system ( $\Delta$ ).

\section{Conclusion}

It is found that the LC2002 SLM can be used for implementing 180 degrees phase-only modulation for phase coded reference beam holographic optical data storage. A near commonpath Mach-Zehnder interferometer was used to measure the phase shift. It is shown experimentally that the transmissivity is more sensitive to the precise orientations of the optical components when the optical system contains one QWP than in the case when two QWPs are utilised in the system. Variation of \pm 2 degrees in the positions of the optical components in the system containing one QWP lead to maximum $12 \%$ in transmission and 6 degree in phase shift variation, whereas, the same angular errors in the system containing two QWPs leads to a maximum of $2.5 \%$ variation in transmission and 3 degrees in phase.

\section{Acknowledgement}

Dublin Institute of Technology (DIT) provided financial support for this project. The authors thank Prof. I. Moreno (Universidad Miguel Hemández), Prof. A. Marquez (Univ. de Alicante), Dr. R. Jallapuram(DIT), S. Osten (Holoeye), and Dr. T. Babeva (DIT). 


\section{References:}

[1]. Birendra Bahadur 'Liquid Crystals - Applications and Uses', Vol.01, 1990.

[2]. C. Soutar and K. Lu, 'Determination of the physical properties of an arbitrary twistednematic liquid crystal cell', Opt. Engg. 33, 2704-2712, 1994.

[3]. J. A. Davis, D. B. Allison, K. G. D'Nelly, M. L. Wilson, and. I Moreno, 'Ambiguities in measuring the physical parameters for twisted-nematic liquid crystal spatial light modulators', Opt. Eng. 38(4), 705-709, 1999.

[4]. Jeffrey A. Davis, Phitbert Tsai, Kevin G. D'Nelly, Ignacio Moreno, 'Simple technique for determining the extraordinary axis direction for twisted-nematic liquid crystal spatial light modulators', Opt. Eng. 38(4) 705-709, 1999.

[5]. A. Marquez, J. Campos, M.J.Yzuel, I. Moreno, J. Davis, C. Lemmi, A. Moreno, A. Robert 'Characterization of edge effects in twisted nematic liquid crystal displays', Optical Engg.39(12), 3301-3307, 2000.

[6]. A Marquez, C. Iemmi, I. Moreno, Jeffrey A. Davis, Juan Campos, 'Quantitative prediction of the modulation behavior of twisted nematic liquid crystal displays based on a simple physical model', Opt. Eng. 40(11), 2558-2564, 2001.

[7]. J. A. Davis, P. Tsai, D. M Cottrell, T. Sonehara, and J. Amako, 'Transmission variations in liquid crystal spatial light modulators caused by interference and diffraction effects', Opt. Eng. 38, 1051-1057, 1999.

[8]. Judit Reményi, Péter Várhegyi, László Domján, Pál Koppa, and Emõke Lõrincz 'Amplitude, Phase, and Hybrid Ternary Modulation Modes of a Twisted-Nematic Liquid-Crystal Display at $\sim 400$ nm', Applied Optics, Vol. 42, No.17, 3428-3434, 2003.

[9]. J. A. Davis, I. Moreno, and P. Tsai, 'Polarization eigenstates for twisted-nematic liquid crystal displays,' Appl. Opt. 37, 937-945, 1998.

[10]. Konforti N, Marom E, Wu S.T, 'Phase-Only Modulation with Twisted Nematic LiquidCrystal Spatial Light Modulators', Optics Letters, Vol: 13, Issue: 3, Pages: 251-253, 1988.

[11]. Thomas H. Barnes, Tomoaki Eiju, Kiyofumi Matusda, and Naotake Ooyama, 'Phase -only modulation using a twisted nematic liquid-crystal television', Applied Optics, Vol: 28, Issue: 22 Pages: 4845-4852, 1989.

[12]. C. Soutar and K. Lu, 'Determination of the physical properties of an arbitrary twisted nematic liquid crystal cell', Opt. Eng.33, 2704-2712, 1994. 
[13]. J. Amako and T. Sonehara, 'Kinoform using an electrically controlled birefringent liquid crystal spatial light modulator,' Appl. Opt. 30, 4622-4628, 1991.

[14]. J. C. Kirsch, D. A. Gregory, M. W. Thie, and B. K. Jones, 'Modulation characteristics of the Epson liquid crystal television', Opt. Eng. 31, 963-970, 1992.

[15]. Zhang Z, Lu G and Yu F T S, 'Simple method for measuring phase modulation in liquid crystal televisions', Opt. Eng, 33, 3018-22, 1994.

[16]. McClain J L Jr., Erbach P S, Gregory D A and Yu F T S 'Spatial light modulator phase depth determination from optical diffraction information', Opt. Eng. 35, 951-954, 1996.

[17]. Andrés Márquez Ruiz, $\mathrm{PhD}$ thesis, 'Accurate predictive model for twisted nematic liquid crystal devices. App. for generating programmable apodizers and Fresnel lenses', 57-59, 2001.

[18]. User Manual, 'Phase cam-Phase and Amplitude Measurement', Holoeye, 2005. 